\title{
Transient optical parametric oscillations in resonantly pumped multistable cavity polariton condensates
}

\author{
A. S. Brichkin, ${ }^{1}$ S. G. Tikhodeev, ${ }^{1,2}$ S. S. Gavrilov,${ }^{1}$ N. A. Gippius,,${ }^{2,3}$ S. I. Novikov,${ }^{1}$ A. V. Larionov, ${ }^{1}$ C. Schneider, ${ }^{4}$ \\ M. Kamp, ${ }^{4}$ S. Höfling, ${ }^{4,5}$ and V. D. Kulakovskii ${ }^{1}$ \\ ${ }^{1}$ Institute of Solid State Physics RAS, 142432 Chernogolovka, Russia \\ ${ }^{2}$ A. M. Prokhorov General Physics Institute RAS, Vavilova Street 38, Moscow 119991, Russia \\ ${ }^{3}$ Skolkovo Institute of Science and Technology, Novaya Street 100, Skolkovo 143025, Russia \\ ${ }^{4}$ Technische Physik, Physikalisches Institut and Wilhelm Conrad Röntgen Research Center for Complex Material Systems, \\ Universität Würzburg, D-97074 Würzburg, Germany \\ ${ }^{5}$ SUPA, School of Physics and Astronomy, University of St Andrews, St Andrews KY16 9SS, United Kingdom
}

(Received 31 May 2015; revised manuscript received 7 September 2015; published 30 September 2015)

\begin{abstract}
Transitional processes accompanying switches between steady states in multistable cavity polariton systems are studied experimentally in a low-symmetry high- $Q$ microcavity under resonant optical excitation at normal incidence. We show that the establishment of a high-energy polariton state is influenced by an optical parametric oscillation. Therefore, the emission spectrum reveals the energy-separated signal-idler pairs in both spin components. Accordingly, the time dependencies of the polariton emission exhibit oscillations whose periods correspond to the spectral positions of the scattered states. Thus, the sharp transitions between the steady states of a no-equilibrium polariton condensate enable one to visualize the near-condensate eigenmodes and explore their spectral and spin properties depending on the condensate amplitude and average spin.
\end{abstract}

DOI: 10.1103/PhysRevB.92.125155

PACS number(s): 71.36.+c, 42.65.Pc, 42.55.Sa

\section{INTRODUCTION}

Cavity exciton-polaritons are bosonic quasiparticles resulting from strong quantum well exciton-cavity-photon coupling [1]. Due to the photonic component, polaritons have an extremely small effective mass, while their excitonic component yields efficient polariton-polariton interactions [2-5]. The latter enables thermalization of nonresonantly pumped polaritons and formation of Bose-Einstein condensates in high- $Q$ cavities [6]. Under resonant pumping, the polariton-polariton interaction leads to a variety of nonlinear collective effects such as parametric scattering [7-12], bi- and multistability [12-19], pattern formation [20], self-organization [21-23], and bright polariton solitons [24]. All these phenomena imply strong redistributions of the intracavity field that occur at certain threshold characteristics of excitation.

$\mathrm{Bi}$ - and multistabilities manifest themselves in sharp jumps of the cavity-field intensity and polarization. They are related to the positive feedback loop between the cavity-field intensity and the resonance frequency of the nonlinear polariton mode under pumping above the polariton energy $E_{\mathrm{LP}}$ [12-14]. The blue-shift of the resonance frequency is ensured by a repulsive polariton-polariton interaction. Intermode polariton parametric scattering, also referred to as polariton optical parametric oscillation (OPO), is most pronounced under pumping near the "magic angle." It results in the formation of macro-occupied signal and idler polariton modes in accordance with the phase matching conditions [7-12,25].

Both effects, multistability and parametric scattering, were studied mainly under continuous-wave pumping conditions. However, they attract much interest in view of ultrafast optical switches that proceed on the picosecond scale [26-29] that is comparable to the intrinsic lifetime of polaritons in GaAs-based cavities with $Q$ factor of several thousands. Thus, they have to be studied with appropriate space and time resolutions.
Recent studies have shown that nonequilibrium transitions related to multistability as well as OPO formation are in fact complex processes involving a chain of instabilities and relaxation effects that may have distinct characteristic times. In particular, it has been found theoretically that sharp transitions between steady states in multistable polariton condensates are mediated by intermode parametric scattering [30]. Furthermore, formation of a macro-occupied OPO signal is supplied by a bistable switch and thus cannot be described by an analogy with a second-order phase transition [22,23]. It has also been found that the transitions from the low- to high-energy steady state in a bistable condensate lead to damped oscillations due to the scattering of the externally pumped mode into a signal-idler pair with different frequencies. Thus, although the final high-energy state is single mode, its attainment is accompanied by a transient OPO process [31].

In this work we perform an experimental study of the transient OPO process that occurs immediately after the sharp jump in a resonantly driven multistable cavity polariton condensate. First, we experimentally verify our recent prediction [31] that the jump into a high-energy state in high$Q$ cavities with $Q \gtrsim 4 \times 10^{4}$ is followed by comparatively long-term damped oscillations. This result supplements the previous studies of the dynamics of such transitions [20,32] and may contribute to practical applications in fast optical switches. Second, we demonstrate an experimental approach to studies of near-condensate polariton modes. These modes are excited in the course of a sharp transition between steady states and can be explored depending on the driven mode parameters in situ, i.e., without the use of an additional probe laser. Third, we show that a theoretical approach based on the Bogolyubov approximation in studies of near-condensate states in macro-occupied Bose systems is applicable to description of strongly nonequilibrium polariton condensates with a nonzero occupancy of the transient OPO states. We show that the Bogolyubov approach describes self-consistently the 
frequencies of the near-condensate states depending on the condensate amplitude; consequently, it predicts the frequencies of the damped oscillations accompanying the relaxation process.

Special attention is paid to the spin properties of the nearcondensate polariton states. Their spin and frequency were theoretically calculated [33] using the Bogolyubov approach, yet, so far, to the best of our knowledge, no experimental verifications have been published. In our experiments we employ a low-symmetry polariton system that exhibits a linearto-circular polarization conversion on reaching the threshold density [28]. We show that the damped oscillations of the condensate density are determined by the energy gap between the condensate and the copolarized signal-idler pair. At the same time the oscillations of the condensate polarization (average spin) are determined by the frequencies of the nearcondensate modes that are counterpolarized with respect to the driven (condensate) mode. We conclude that the mean-field approach based on the Gross-Pitaevskii equations provides a self-consistent description of both the density and spin of the resonantly pumped polariton condensate in the course of its multistable switches.

The paper is arranged as follows. The experimental details and the sample are described in Sec. II. The experimental results are described in Sec. III and discussed in Sec. IV. Section V contains concluding remarks.

\section{EXPERIMENT}

The investigated GaAs-based microcavity (MC) was grown by molecular beam epitaxy on a GaAs substrate. It contained four 7-nm-thick GaAs quantum wells separated by $4 \mathrm{~nm} \mathrm{AlAs}$ barriers at antinodes of the electric field in a half- $\lambda$-thick AlAs cavity. The Rabi splitting was $10.5 \mathrm{meV}$. The large number of layers in the top (bottom) mirror consisting of 32 (36) $\mathrm{Al}_{0.2} \mathrm{Ga}_{0.8} \mathrm{As} / \mathrm{AlAs}$ Bragg reflectors resulted in a strong suppression of the polariton leakage via the photonic component and, hence, in a high-quality factor ( $Q$ factor), and hence, in a strong suppression of the polariton leakage. To perform transmission measurements we locally $(\sim 300 \times 700$ $\mu \mathrm{m})$ removed the GaAs substrate with the use of citric $\mathrm{acid} /$ hydrogen peroxide selective etch (AlAs in the nearest mirror served as the etch stop layer). The MC layer in the etched area was slightly strained due the small difference in the lattice parameters of GaAs and AlAs, which caused lowering of the lateral symmetry and, as a consequence, a small (30-140 $\mu \mathrm{eV}$ ) splitting of the low polariton (LP) states varying in the etched region.

To avoid excitation of the exciton reservoir under resonant excitation of the polaritons at the LP branch we investigated the $\mathrm{MC}$ with a negative cavity-mode-exciton detuning $E_{C}-$ $E_{X} \approx-6.5 \mathrm{meV}$ at zero in-plane wave vector $\mathbf{k}=\mathbf{0}$. The LP energies $E_{\mathrm{LP}}(k=0)$ were determined from the transmission spectra recorded in a linear regime using weak 1.5-ps-long pulses (spectral width $\sim 2 \mathrm{meV}$ ) from a Ti:sapphire laser with a repetition frequency of $80 \mathrm{MHz}$. The spectra exhibited two cross-linearly polarized lines $\mathrm{LP}_{x}$ and $\mathrm{LP}_{y}$ separated by $\delta_{x y}=E_{\mathrm{LP}, x}-E_{\mathrm{LP}, y}=0.08 \pm 0.01 \mathrm{meV}$. The eigenstates were polarized along the sides of the etched window. The decay rate of the LPs at $k=0$ was determined from the time-resolved measurements of the time-dependent polariton emission under resonant excitation with 1.5-ps-long pulses with a laser beam spot of $30 \mu \mathrm{m}$. The polariton lifetime $\tau$ was found to vary over the MC plane in the range of 15-25 ps, which corresponded to the $Q$-factor range of $\sim(3-5) \times 10^{4}$.

The sample was placed into an optical cryostat at $T=6 \mathrm{~K}$ and excited by nearly transform-limited optical pulses with a repetition rate of $8 \mathrm{MHz}$ and duration of 80 ps generated by a mode-locked Ti:sapphire laser directed along the cavity normal. The pump beam was focused into a 30- $\mu \mathrm{m}$-wide spot on the sample. The pump energy $\hbar \omega_{p}$ was $0.2-0.3$ $\mathrm{meV}$ above the LP resonance and the pump polarization was close to linear: its degrees of linear and circular polarizations were $\rho_{x, y}=\left(P_{x}-P_{y}\right) /\left(P_{x}+P_{y}\right)=+0.998$ and $\rho_{c}=$ $\left(P^{+}-P^{-}\right) /\left(P^{+}+P^{-}\right) \approx 0.05$, respectively, where $P_{x, y}$ and $P^{+,-}$are the pump intensities in the linear $\left(\pi_{x, y}\right)$ and circular $\left(\sigma^{ \pm}\right)$polarizations. A polarizer and a $\lambda / 4$ wave plate were employed to select a particular polarization component.

The emission signal was recorded in transmission geometry from the back side of the sample to avoid the contribution from the scattered laser beam. The dynamics of the emission intensity was detected by a streak camera from the central $\left(\sim 4 \times 4 \mu \mathrm{m}^{2}\right)$ part of the excited spot with time resolution of 6 ps. For spectral analysis of the LP emission, the spectra were recorded with a resolution as high as $0.07 \mathrm{meV}$, which limited the time resolution by $\sim 50 \mathrm{ps}$.

\section{EXPERIMENTAL RESULTS}

We study the transitional processes in a multistable LP system in laterally strained MCs excited with spectrally narrow 80-ps-long pulses at $\hbar \omega_{p}=E_{\mathrm{LP}}(k=0)+\Delta$ where $\Delta=$ $0.22 \pm 0.02 \mathrm{meV} \gg \delta_{x y}=0.08 \mathrm{meV}$. The pump polarization is close to that of the upper ( $\pi_{x}$ polarized) LP sublevel. The photoexcited LP system in this case demonstrates a multistable behavior [15]. The calculated dependence of the steady-state polariton amplitude on the external pump is shown in Fig. 1. It has three stability branches with different polarizations: the LP polarization on the lower and upper branches coincides with that of the pump, while it is close to circular on the middle

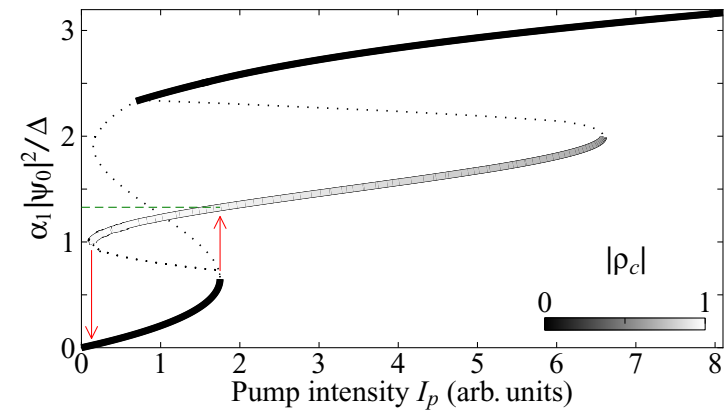

FIG. 1. (Color online) Steady-state response function calculated within the one-mode approximation for a low-symmetry MC excited with the light whose linear polarization coincides with that of the upper LP level. Thin dotted lines show asymptotically unstable solutions. Red arrows indicate the $\pi$-to- $\sigma$ and reverse transitions that are considered in this work. 

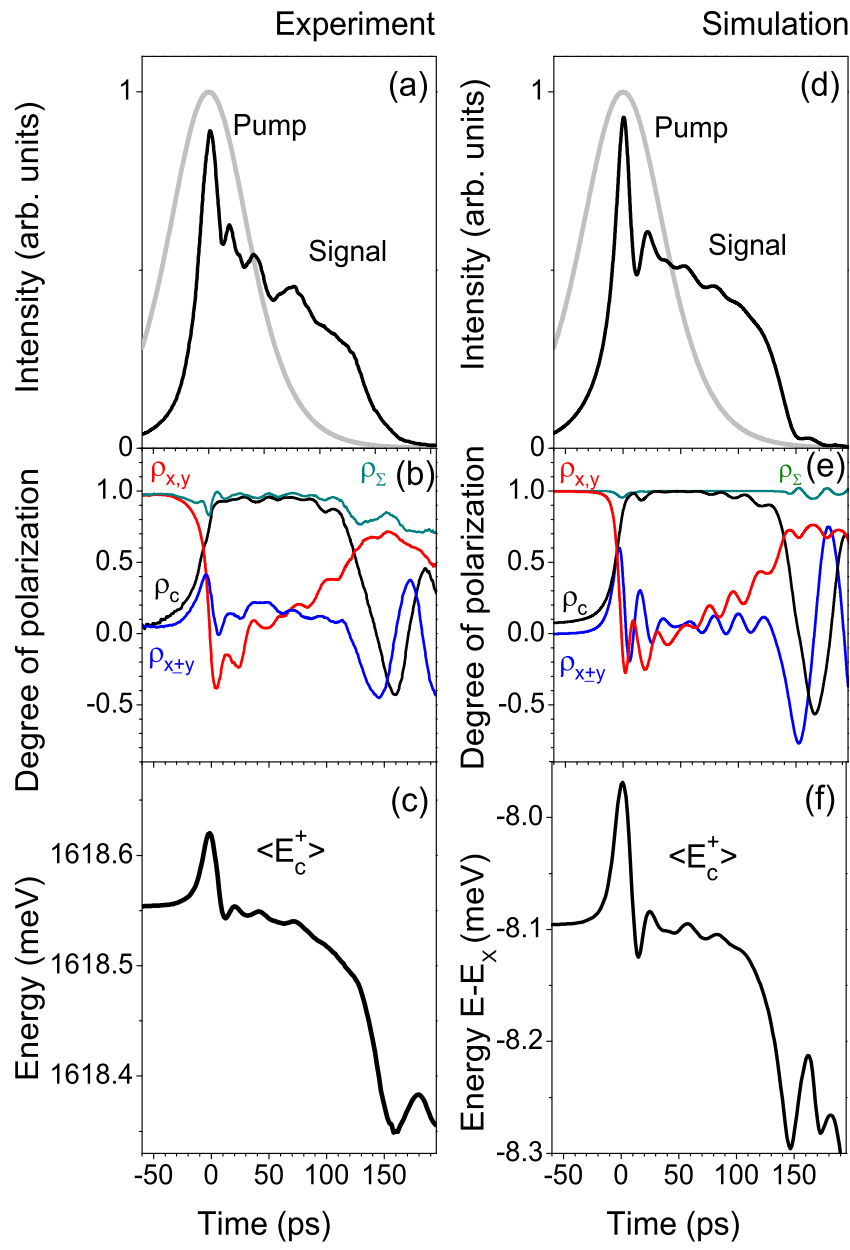

FIG. 2. (Color online) Time dependencies of condensate emission intensity $I_{c}[(\mathrm{a}),(\mathrm{d})]$, degrees of polarization $\rho_{c}, \rho_{x, y}, \rho_{x \pm y}$, integral polarization degree $\rho_{\Sigma}[(\mathrm{b}),(\mathrm{e})]$, and transition energy [(c),(f)]. The gray lines in (a) and (d) show the pump line shape. Left and right panels show the experimental and calculated dependencies, respectively.

branch so that the transition from the lower to middle branch proceeds with polarization switching [28].

Figures 2(a) and 2(b) show the time dependencies of the emission intensity, $I_{c}$, and the degrees of the circular $\left(\rho_{c}\right)$, linear $\left(\rho_{x, y}\right)$, and diagonal $\left(\rho_{x \pm y}\right)$ polarizations of the LP system recorded with a time resolution of 4 ps in the case when the peak pump power $\sim 500 \mathrm{~kW} / \mathrm{cm}^{2}$ over the excited spot diameter of $30 \mu \mathrm{m}$ is set slightly above the threshold for transition from the lower to middle stability branch; thus, the transition takes place near the pulse maximum. The pump shape is shown in Fig. 2(a) by a thick gray line; time $t=0$ corresponds to the pulse maximum. The pump polarization is close to linear: $\rho_{x, y}=0.99$ and $\rho_{c}=0.05$. The deviation is necessary to obtain reproducible circular polarization on the middle stability branch.

Figures 2(a) and 2(b) show that the threshold increase in $I_{c}$ at $t \approx-20 \mathrm{ps}$ is followed by a sharp change in the signal polarization from nearly linear to nearly circular in accordance with the steady-state response function shown in Fig. 1. Further, Fig. 2(a) shows that the transition is followed by a well pronounced decaying oscillation in the emission intensity around its quasistationary value on the $\sigma$ branch. The oscillation period $T_{\text {int }}$ increases gradually from $\approx 20 \mathrm{ps}$ to $\approx 30 \mathrm{ps}$ at $t>80 \mathrm{ps}$.

Figure 2(b) shows that the total degree of polarization $\rho_{\Sigma}=\sqrt{\rho_{c}^{2}+\rho_{x, y}^{2}+\rho_{x \pm y}^{2}}$ at $0<t<110$ ps exceeds 0.96 throughout the period when the condensate stays on the $\sigma$ branch, whereas the linear and circular degrees of polarization show pronounced oscillations. The oscillation periods coincide in different polarizations and are time independent within the experimental error, $T_{\mathrm{pol}}=21 \pm 2 \mathrm{ps}$.

The oscillations in $I_{c}$ and $\rho_{i}$ point out that the polariton system contains several coherent modes. The oscillation period $T \approx 20 \mathrm{ps}$ corresponds to the energy gap of $0.2 \mathrm{meV}$. To resolve the modes the spectra are recorded with a high spectral resolution of $\Delta \hbar \omega=0.07 \mathrm{meV}$. Figures 3(a)-3(e) show the spectra at five reference time points corresponding, respectively, to (i) the system on the lower $\pi$ branch ( $t=$ $-50 \mathrm{ps})$, (ii) its transition from the $\pi$ to $\sigma \operatorname{branch}(t=-10 \mathrm{ps})$, (iii) its relaxation to the quasistationary state on the $\sigma$ branch
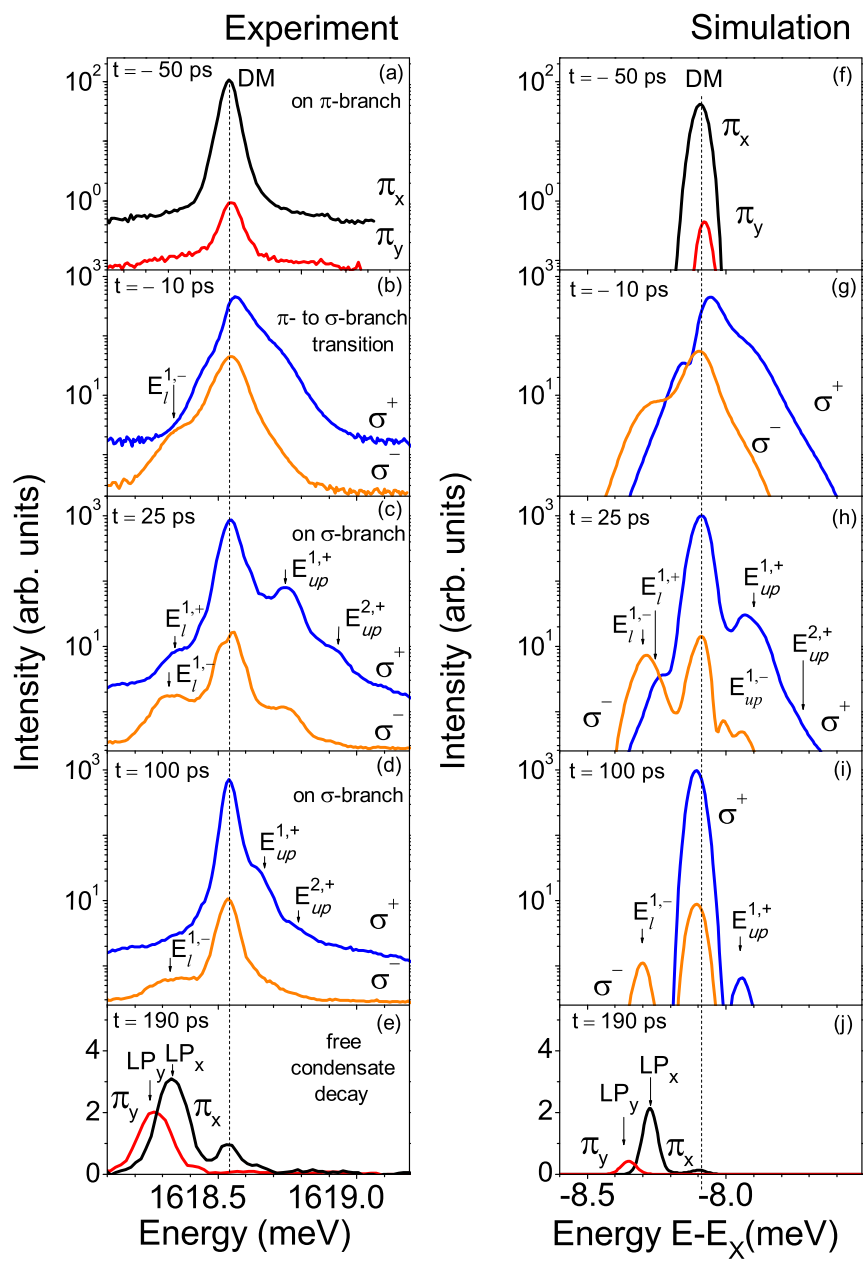

FIG. 3. (Color online) Experimental (left panel) and calculated (right panel) $\sigma^{+}$and $\sigma^{-}$polarized emission spectra of resonantly pumped LP condensate at several reference time moments that are indicated in (a)-(j) in the upper left corner. The background in the experimental spectra at the intensity level of $\sim 1 / 300 I_{\mathrm{DM}}$ is due to the presence of scattered light in the spectrometer. 
( $t=25$ and $90 \mathrm{ps}$ ), and (iv) its free decay after leaving the $\sigma$ branch $(t=200 \mathrm{ps})$. It should be emphasized that the high spectral resolution limits the time resolution down to $\sim 50 \mathrm{ps}$, i.e., the emission spectra are averaged over the time nearly twice as long as the oscillation period.

Figure 3 shows that the emission spectrum at $t=-50 \mathrm{ps}$ (the regime of weak nonlinearity) consists of a single spectrally narrow and linearly polarized line corresponding to the driven mode (DM) at $\hbar \omega_{p}$. The sharp increase in the cavity field near the transition to the $\sigma$ branch $(t \sim-10 \mathrm{ps})$ is followed by its strong spectral broadening as it is expected from the Fourier transform. Note, however, that the line broadening is (i) strongly asymmetric and (ii) different in the $\sigma^{+}$and $\sigma^{-}$ polarizations, which is indicative of the resonance states near the driven mode although there are no nearby resonances in this range in the unexcited MC.

Figure 3(c) shows that the broad tails of the DM line transform with time into several weak components labeled as $E_{l \text { (up) }}^{i,+(-)}$. Here the low indices $l$ (up) correspond to the modes below (above) the DM, respectively. The upper indices + and - indicate the sign of their dominating circular polarization. The strongest satellite component in the weak $\left(\sigma^{-}\right)$polarization, $E_{l}^{1,-}$, is at the red (Stokes) side of the DM. On the contrary, the strongest satellite mode in the dominated $\sigma^{+}$polarization, $E_{\text {up }}^{1,+}$, is at the blue (anti-Stokes) side of the DM line. At $t=25 \mathrm{ps}$ its intensity is about ten times smaller than that of the DM one. The components $E_{\text {up }}^{1,+}$ and $E_{l}^{1,+}$ are symmetric relative to the DM line; the gap $\delta^{+}$is $0.20 \pm 0.02$ $\mathrm{meV}$. One more line in the $\sigma^{+}$spectrum, $E_{\text {up }}^{2,+}$, is separated by $2 \delta^{+}$from the DM line. We attribute it to the four-wave mixing of the DM and $E_{\text {up }}^{1,+}$, taking into account (i) the equality of the gaps between the $E_{\text {up }}^{2,+}$ and $E_{\text {up }}^{1,+}$ lines and the $E_{\text {up }}^{1,+}$ lines and the DM, and (ii) the proportionality of the intensity of the $E_{\text {up }}^{2,+}$ line to the product of the squared intensity of the DM line and the intensity of the $E_{\text {up }}^{1,+}$ line. Similar satellites have been earlier observed and investigated in the studies of stimulated parametric polariton-polariton scattering in MCs excited under the magic angle $[9,34,35]$.

Comparison of Figs. 3(b)-3(d) shows that the satellite lines decay much faster than the driven mode, thus the parametric scattering is over soon after the system jumps on the $\sigma$ branch, which is in agreement with the theoretical predictions. It is also seen that the gap between the DM and $E_{l, \text { (up) }}^{+}$decreases with time (down to $\delta^{+}=0.15 \pm 0.03 \mathrm{meV}$ at $t=80 \mathrm{ps}$ ), whereas the gap between DM and $E_{l, \text { (up) }}^{-}$remains nearly constant, $\delta^{-}=$ $0.21 \pm 0.02 \mathrm{meV}$.

Finally, Fig. 3(e) shows the emission spectra of the freely decaying polariton system after termination of the pump pulse. The decrease in the LP density and interparticle interaction results in recovery of the initial cross-linearly polarized sublevels $\mathrm{LP}_{x}$ and $\mathrm{LP}_{y}$. As a result, the spectra consist of two cross-linearly polarized lines whose polarizations and spectral positions are the same as in an empty cavity. A more detailed consideration of the condensate behavior at this stage can be found in Ref. [32].

Thus, the sharp transition from the lower to middle stability branch is followed by the appearance of (i) new macro-occupied signal-idler pairs and (ii) oscillations in the condensate intensity and polarization. The oscillation period of $I_{c}$ is determined by the spectral gap between the DM and the copolarized signal-idler modes. At the same time the period of the polarization oscillations corresponds to the gap between the DM and the signal-idler modes with opposite circular polarizations. Note as well that the beating of the DM and the copolarized signal-idler modes causing the condensate density oscillation should give rise to transient oscillations of the spectral "center of mass" $\left\langle E_{c}^{+}(t)\right\rangle=\int \hbar \omega I_{c}(\omega, t) / \int I_{c}(\omega, t) d \omega$ of the condensate emission in the spectra recorded with a time resolution adjusted to the time scale of the process in question. The oscillation period of the intensity is $\hbar / \delta^{+} \sim 20 \mathrm{ps}$. In order to measure the variations in $\left\langle E_{c}^{+}(t)\right\rangle$, the emission spectra were recorded with a 6 -ps time resolution. The extracted dependence $\left\langle E_{c}^{+}(t)\right\rangle$ is displayed in Fig. 2(c). It is clearly seen that $\left\langle E_{c}^{+}(t)\right\rangle$ shows a well pronounced decaying oscillation similar to that of $I_{c}(t)$.

\section{DISCUSSION}

The frequencies of the near-condensate resonances in a nonequilibrium quasiresonantly pumped LP system can be calculated within the framework of the Gross-Pitaevskii equations using the linearization procedure. In contrast to the well-known Bogolyubov solution for an equilibrium Bose condensate with an acoustic spectrum of excitations [36,37], the $k=0$ resonances in the driven condensate are separated from DM by finite energy gaps and appear both above and below the DM line. In a symmetric MC with circularly (linearly) polarized DM there are two pairs of signal-idler resonances with opposite polarizations $\sigma^{+}$and $\sigma^{-}\left(\pi_{x}\right.$ and $\pi_{y}$ ) [33]. In the case of $\sigma$-polarized excitation the parametric scattering is allowed only during bistable transitions between the stability branches and occurs only into the copolarized states [31].

Figure 3 shows that the jump of the LP system taking place in the MC of lowered symmetry from the lower $(\pi)$ to middle $(\sigma)$ stability branch is followed by the appearance of two signal-idler pairs, one of which is $\sigma^{+}$and the other $\sigma^{-}$polarized. Their close-to-circular polarizations are a direct consequence of the circular polarization of the driven mode.

In the coherent mean-field approach, the spinor GrossPitaevskii equations for a single LP mode driven by a coherent pump with $k=0$ can be written as

$$
\begin{aligned}
i \hbar \frac{\partial \psi_{+}}{\partial t}= & \left(E_{\mathrm{LP}}-i \hbar / \tau-\frac{\hbar^{2} \nabla^{2}}{2 m}\right) \psi_{+}+\frac{\delta_{x y}}{2} \psi_{-} \\
& +\left(\alpha_{1}\left|\psi_{+}\right|^{2}+\alpha_{2}\left|\psi_{-}\right|^{2}\right) \psi_{+}+F_{+}(t) e^{-i \omega_{p} t}, \\
i \hbar \frac{\partial \psi_{-}}{\partial t}= & \left(E_{\mathrm{LP}}-i \hbar / \tau-\frac{\hbar^{2} \nabla^{2}}{2 m}\right) \psi_{-}+\frac{\delta_{x y}}{2} \psi_{+} \\
& +\left(\alpha_{2}\left|\psi_{+}\right|^{2}+\alpha_{1}\left|\psi_{-}\right|^{2}\right) \psi_{-}+F_{-}(t) e^{-i \omega_{p} t},
\end{aligned}
$$

where $\psi$ is the macroscopic wave function and $F(t)$ is the pump amplitude, $\alpha_{1,2}$ are the polariton-polariton interaction constants for LPs with the same and opposite spin projections, and $\tau$ is the polariton decay time.

The linearization of Eqs. (1) and (2) is made [33] assuming a solution in the form of a sum of the driven condensate modes 
(oscillating with pump frequency) and weak near-condensate modes oscillating with frequencies $E_{s}^{\sigma} / \hbar$ (signal) and $E_{i}^{\sigma} / \hbar=$ $2 \omega_{p}-E_{s}^{\sigma *} / \hbar$ (idler),

$$
\psi_{\sigma}=\Psi_{\sigma}+\delta \psi_{\sigma}=A_{\sigma} e^{-i \omega_{p} t}+B_{\sigma} e^{-i E_{s}^{\sigma} t / \hbar}+C_{\sigma} e^{-i E_{i}^{\sigma} t / \hbar}
$$

where $A_{\sigma}, B_{\sigma}$, and $C_{\sigma}$ are the amplitudes of the driven, signal, and idler modes, respectively, and $\sigma=+,-$. The multistable dependence of condensate intensity and polarization on pump intensity and polarization was investigated in detail in Ref. [15]; here we are interested in the properties of the near-condensate modes [33]. The resulting linearized equations for the signal and idler amplitudes can be written as

$$
\left(\begin{array}{cc}
H_{\sigma \sigma^{\prime}, s s}-E_{s}^{\sigma} \delta_{\sigma \sigma^{\prime}} & H_{\sigma \sigma^{\prime}, s i} \\
-H_{\sigma \sigma^{\prime}, s i}^{*} & \left(2 \hbar \omega_{p}-E_{s}^{\sigma}\right) \delta_{\sigma \sigma^{\prime}}-H_{\sigma \sigma^{\prime}, s s}^{*}
\end{array}\right)\left(\begin{array}{l}
B_{\sigma^{\prime}} \\
C_{\sigma^{\prime}}^{*}
\end{array}\right)=0
$$

where

$$
\begin{gathered}
H_{\sigma \sigma^{\prime}, s s}=H_{\sigma \sigma^{\prime}}^{0}+\left(\begin{array}{cc}
2 \alpha_{1} A_{+} A_{+}^{*}+\alpha_{2} A_{-} A_{-}^{*} & \alpha_{2} A_{+} A_{-}^{*} \\
\alpha_{2} A_{-} A_{+}^{*} & 2 \alpha_{1} A_{-} A_{-}^{*}+\alpha_{2} A_{+} A_{+}^{*}
\end{array}\right), \\
H_{\sigma \sigma^{\prime}, s i}=\left(\begin{array}{cc}
\alpha_{1} A_{+} A_{+} & \alpha_{2} A_{+} A_{-} \\
\alpha_{2} A_{-} A_{+}+ & \alpha_{1} A_{-} A_{-}
\end{array}\right),
\end{gathered}
$$

and

$$
H_{\sigma \sigma^{\prime}}^{0}=\left(\begin{array}{cc}
E_{\mathrm{LP}}-i \hbar / \tau-\hbar \omega_{p} & \delta_{x y} / 2 \\
\delta_{x y} / 2 & E_{\mathrm{LP}}-i \hbar / \tau-\hbar \omega_{p}
\end{array}\right) .
$$

In the case of $\sigma^{+}$polarization of the DM on the $\sigma$-stability branch $\left|A_{+}\right| \gg\left|A_{-}\right|$. Assuming $\alpha_{1} \gg\left|\alpha_{2}\right|$, Eqs. (5) and (6) take a simplified form,

$$
H_{\sigma \sigma^{\prime}, s s}=H_{\sigma \sigma^{\prime}}^{0}+\left(\begin{array}{cc}
2 \alpha_{1} A_{+} A_{+}^{*} & 0 \\
0 & \alpha_{2} A_{+} A_{+}^{*}
\end{array}\right)
$$

and

$$
H_{\sigma \sigma^{\prime}, s i}=\left(\begin{array}{cc}
\alpha_{1} A_{+} A_{+} & 0 \\
0 & 0
\end{array}\right)
$$

Finally, taking into account the smallness of the LP splitting $\left(\delta_{x y} \ll \Delta\right)$, we see that (i) the equations for the $\sigma^{+}$signal and idler coincide with those in the case of the purely circular pump in symmetric MCs [31], and (ii) the $\sigma^{-}$signal and idler decouple. Then the signal and idler energies become

$$
\begin{gathered}
E_{s, i}^{+}=E_{\mathrm{up}, l}^{+}=\hbar \omega_{p} \pm \sqrt{\left(2 \alpha_{1} n-\Delta\right)^{2}-\left(\alpha_{1} n\right)^{2}}-i \hbar / \tau \\
E_{s}^{-}=E_{l}^{-}=E_{\mathrm{LP}}+\alpha_{2} n-i \hbar / \tau \\
E_{i}^{-}=E_{\mathrm{up}}^{-}=E_{\mathrm{LP}}+2 \Delta-\alpha_{2} n-i \hbar / \tau
\end{gathered}
$$

where $n \sim n_{+}=A_{+} A_{+}^{*}$ is the condensate density.

We consider the polariton system on the $\sigma^{+}$stability branch where the polariton-polariton interaction energy $\alpha_{1} n>\Delta$. Formula (11) shows that the $\sigma^{-}$signal is located below the DM (Stokes satellite) exactly at the resonance frequency of the $\sigma^{-}$component in the two-component spinor polariton system with a strongly dominating $\sigma^{+}$polarization. The behavior of the $\sigma^{+}$signal-idler pair is different. First, the $\sigma^{+}$signal is located above the DM (anti-Stokes satellite), which conforms to the condition $\alpha_{1} n>\Delta$. Moreover, it follows from Eq. (10) that $E_{s}^{+}-E_{\mathrm{LP}}$ exceeds markedly the polariton-polariton interaction energy $\alpha_{1} n$.
The value of $\alpha_{1} n$ can be estimated from the $S$-shaped curve in Fig. 1 calculated for the investigated MC with parameters $\Delta=0.22 \mathrm{meV}$ and $\delta_{x y}=0.08 \mu \mathrm{eV}$. From Fig. 1 it is seen that the jump occurs into the $\sigma$-polarized state with $\alpha_{1} n \approx 1.33 \Delta$ (see the right vertical arrow). The substitution of this value to formula (10) gives $\delta^{+}=\left|\operatorname{Re} E_{\mathrm{up}(l)}^{+}-\hbar \omega_{p}\right| \approx \Delta=0.22 \mathrm{meV}$, which is in good agreement with the experimental value, $\delta^{+}=$ $0.20 \pm 0.02 \mathrm{meV}$ at $t=25 \mathrm{ps}$. Further, from Eqs. (10)-(12) it is seen that the decrease in the condensate density leads to the pronounced decrease in $\delta^{+}$, which is also in agreement with the experiment: Figure 3 shows that $\delta^{+}$decreases down to $\sim 0.15 \mathrm{meV}$ at $t=80 \mathrm{ps}$.

The behavior of $\delta^{-}$is quite different. Its experimental value is $0.21 \pm 0.02 \mathrm{meV}$ at $t=25 \mathrm{ps}$, and it remains constant within the experimental error during the excitation pulse. This value coincides approximately with $\Delta=0.22 \pm 0.02$ meV. The latter, according to Eq. (11), means that $\alpha_{2}$ is very small, $\left|\alpha_{2}\right| / \alpha_{1} \lesssim 0.1$, which is in agreement with the earlier estimations of $\alpha_{2}$ for the MC with a negative detuning [4].

The results of more detailed numerical simulations of the dynamics of the driven condensate within the framework of spinor Gross-Pitaevskii equations (1) and (2) are shown in the right panels of Figs. 2 and 3. The parameters $E_{\mathrm{LP}}, \omega_{p}$, and pump pulse shape $F(t)$ are taken from the experiment. The amplitude of $F$ and the LP decay time $\tau$ are found from adjustment of the time moments corresponding to the jumps from the $\pi$ to $\sigma$ branch and back, respectively. The ratio $\alpha_{2} / \alpha_{1}=-0.1$ was chosen to describe the spectral position of the line $E_{l}^{1,-}$, as explained above. The found value $\tau=25 \mathrm{ps}$ coincides with the decay time of the freely decaying polariton system measured at $t>150 \mathrm{ps,} \mathrm{which} \mathrm{confirms} \mathrm{its} \mathrm{validity.}$

Figure 2 shows that the simulations reproduce the transient damped-oscillation behavior of both the condensate field and its polarization. The oscillation periods in the emission intensity, $T_{\mathrm{int}}$, and polarization, $T_{\mathrm{pol}}$, are connected mainly to the gaps between the $\sigma^{+}$DM and $\sigma^{+}$(i.e., $\delta^{+}$) and $\sigma^{-}$(i.e., $\delta^{-}$) near-condensate modes, respectively. The calculated value of $T_{\text {pol }}$ coincides with the experimental one $(21 \pm 2 \mathrm{ps})$. The 
calculated period $T_{\text {int }}$ increases with time slightly slower than in the experiment, which correlates with a slower decrease in the calculated condensate density compared to the experiment. The beating of the DM and near-condensate modes also causes a transient decaying oscillation of the center of the mass of the condensate emission spectrum recorded with a high time resolution as shown in Figs. 2(c) (experiment) and 2(f) (simulations).

The calculated emission spectra have been obtained from the frequency-domain analysis of the calculated dependencies $\psi(t)$ by performing Fourier transform over 50-ps time intervals. They are shown in the right panel of Fig. 3. Comparison of the experimental spectra with the calculated ones shows that the latter reproduce all the main features: (i) a highly asymmetric and different in two polarizations line broadening during the transition from the $\pi$ to $\sigma$ branch at $t \sim 0$, (ii) the spectral positions of the signal and idler modes in both polarizations, and (iii) the difference in the signal and idler intensities.

It is worthwhile to note that the $\sigma^{+}$polarized signal and idler are not decoupled and thereby cannot be considered as independent quasiparticles. In spite of the fact that $\omega_{s}+\omega_{i}=$ $2 \omega_{p}$ (that is merely a phase matching condition), the parametric scattering is not the scattering of two LPs from the driven mode into one-signal and one-idler quasiparticles. Indeed, the signal-to-idler ratio is not equal to 1 and actually controlled by the condensate density, it tends to 1 only at $\alpha_{1} n / \Delta \rightarrow 1$. Underline that the observed large difference in the signal and idler intensities of about five times cannot be explained by a possible difference in the excitonic fraction of the signal and idler states. Indeed, the latter is very small due to the smallness of the energy detuning between these states $(\sim 0.3$ $\mathrm{meV}$ ) compared to Rabi splitting (10.5 meV).

In the approximation of weak signal and idler intensities the intensity ratio $I\left(E_{l}^{1,+}\right) / I\left(E_{\mathrm{up}}^{1,+}\right)$ can be estimated from the linearized equations (4), (8), and (9) (see also Ref. [31]). In this approximation it is determined by the dimensionless parameter $x=\alpha_{1} n / \Delta$ :

$$
I\left(E_{l}^{1,+}\right) / I\left(E_{\text {up }}^{1,+}\right)=\left(2 x-1-\sqrt{1-4 x+3 x^{2}}\right)^{2} / x^{2} .
$$

At $\alpha_{1} n>\Delta$ the anti-Stokes component is more intensive than the Stokes one, which is in qualitative agreement with the experiment. The experimental ratio $I\left(E_{l}^{1,+}\right) / I\left(E_{\mathrm{up}}^{1,+}\right) \sim 1 / 8$ at $t=25 \mathrm{ps}$ is about twice smaller than that estimated from Eq. (13) in the limit of very small intensities of the signalidler modes compared to the driven one. The account for the nonlinearity leads to a further decrease of this ratio as is seen from the simulation results in Fig. 3.

As to the $\sigma^{-}$idler-to-signal ratio, in the above approximation it is uncertain as the $\sigma^{-}$signal and idler are decoupled as long as the DM is $\sigma^{+}$polarized. A very weak relative intensity of the $\sigma^{-}$idler obtained in simulations, is also in agreement with the experiment.

\section{CONCLUSION}

We have investigated the transitional processes accompanying switches between the $\pi$ and $\sigma$ steady states in a multistable polariton system in a low-symmetry high- $Q \mathrm{MC}$. We have shown that the switch is accompanied by a transient optical parametric oscillation resulting in formation of gradually decaying $\sigma^{+}$and $\sigma^{-}$polarized signal-idler pairs. These pairs are observed in the emission spectra in the frequency domain. The beatings of the signal-idler and driven modes are seen as oscillations in the time domain.

The spinor Gross-Pitaevskii equations are found to describe the investigated transitional processes. They reproduce the energies of the signal-idler modes, their polarizations, relative intensities, and decay times as well as transient oscillations in the condensate density and polarization due to intermode beating in the nonequilibrium cavity polariton condensate resonantly generated by laser pulses.

The signal and idler modes copolarized to a driven one are not decoupled. The ratio of their intensities is determined by the ratio $\alpha_{1} n / \Delta$, being close to 1 only when $\alpha_{1} n \rightarrow \Delta$. These modes disappear with pump switching off. By contrast, the signal and idler modes with a circular polarization opposite to that of the DM are decoupled. The signal mode is located at its resonance $E_{s}=E_{\mathrm{LP}}+\alpha_{2} n$ and does not disappear when the pump is switched off.

The oscillation period of the field magnitude $T_{\text {int }}$ is mainly determined by the gap $\delta^{+}$between the copolarized driven and signal modes. This gap is controlled by the strong repulsive interaction in the dense driven mode, which results in a high nonlinearity in $T_{\text {int }}$. In contrast, the oscillation period of condensate polarization $T_{\mathrm{pol}}$ is determined by the difference $\delta^{-}$between the frequency of the driven mode and that of the polariton resonance with an opposite circular polarization. This difference is controlled by the weak interaction of LPs with opposite spin projections, which results in a weak dependence of $T_{\text {pol }}$ on the driven mode density.

Finally, in this work we have shown that switches between steady polariton states in high- $Q$ MCs end up with a comparatively long relaxation process related to the transient oscillations due to OPO modes. This result may find use in the area of fast optical switches based on resonantly driven polariton condensates.

\section{ACKNOWLEDGMENTS}

We thank M. M. Glazov, L. V. Keldysh, N. S. Maslova, and V. B. Timofeev for fruitful discussions. The work was supported by the Russian Science Foundation (Grant No. 1412-01372) and the State of Bavaria.
[1] C. Weisbuch, M. Nishioka, A. Ishikawa, and Y. Arakawa, Phys. Rev. Lett. 69, 3314 (1992).
[2] C. Ciuti, V. Savona, C. Piermarocchi, A. Quattropani, and P. Schwendimann, Phys. Rev. B 58, 7926 (1998). 
[3] P. Renucci, T. Amand, X. Marie, P. Senellart, J. Bloch, B. Sermage, and K. V. Kavokin, Phys. Rev. B 72, 075317 (2005).

[4] M. Vladimirova, S. Cronenberger, D. Scalbert, K. V. Kavokin, A. Miard, A. Lemaître, J. Bloch, D. Solnyshkov, G. Malpuech, and A. V. Kavokin, Phys. Rev. B 82, 075301 (2010).

[5] A. V. Sekretenko, S. S. Gavrilov, and V. D. Kulakovskii, Phys. Rev. B 88, 195302 (2013).

[6] J. Kasprzak, M. Richard, S. Kundermann, F. Baas, P. Jeambrun, J. M. J. Keeling, F. M. Marchetti, M. H. Szymanska, R. André, J. L. Staehli, V. Savonna, P. B. Littlewood, B. Deaveaud, and L. S. Dang, Nature (London) 443, 409 (2006).

[7] P. G. Savvidis, J. J. Baumberg, R. M. Stevenson, M. S. Skolnick, D. M. Whittaker, and J. S. Roberts, Phys. Rev. Lett. 84, 1547 (2000).

[8] R. M. Stevenson, V. N. Astratov, M. S. Skolnick, D. M. Whittaker, M. Emam-Ismail, A. I. Tartakovskii, P. G. Savvidis, J. J. Baumberg, and J. S. Roberts, Phys. Rev. Lett. 85, 3680 (2000).

[9] C. Ciuti, P. Schwendimann, and A. Quattropani, Phys. Rev. B 63, 041303(R) (2001).

[10] D. M. Whittaker, Phys. Rev. B 63, 193305 (2001).

[11] V. D. Kulakovskii, A. I. Tartakovskii, D. N. Krizhanovskii, N. A. Gippius, M. S. Skolnick, and J. S. Roberts, Nanotechnology 12, 475 (2001).

[12] N. A. Gippius, S. G. Tikhodeev, V. D. Kulakovskii, D. N. Krizhanovskii, and A. I. Tartakovskii, Europhys. Lett. 67, 997 (2004).

[13] A. Baas, J.-Ph. Karr, H. Eleuch, and E. Giacobino, Phys. Rev. A 69, 023809 (2004).

[14] N. A. Gippius, I. A. Shelykh, D. D. Solnyshkov, S. S. Gavrilov, Y. G. Rubo, A. V. Kavokin, S. G. Tikhodeev, and G. Malpuech, Phys. Rev. Lett. 98, 236401 (2007).

[15] S. S. Gavrilov, N. A. Gippius, S. G. Tikhodeev, and V. D. Kulakovskii, J. Exp. Theor. Phys. 110, 825 (2010).

[16] T. K. Paraïso, M. Wouters, Y. Léger, F. Morier-Genoud, and B. Deveaud-Plédran, Nat. Mater. 9, 655 (2010).

[17] D. Sarkar, S. S. Gavrilov, M. Sich, J. H. Quilter, R. A. Bradley, N. A. Gippius, K. Guda, V. D. Kulakovskii, M. S. Skolnick, and D. N. Krizhanovskii, Phys. Rev. Lett. 105, 216402 (2010).

[18] C. Adrados, A. Amo, T. C. H. Liew, R. Hivet, R. Houdré, E. Giacobino, A. V. Kavokin, and A. Bramati, Phys. Rev. Lett. 105, 216403 (2010).

[19] S. S. Gavrilov, A. S. Brichkin, A. A. Dorodnyi, S. G. Tikhodeev, N. A. Gippius, and V. D. Kulakovskii, JETP Lett. 92, 171 (2010).
[20] A. V. Sekretenko, S. S. Gavrilov, S. I. Novikov, V. D. Kulakovskii, S. Höfling, C. Schneider, M. Kamp, and A. Forchel, Phys. Rev. B 88, 205302 (2013).

[21] S. S. Gavrilov, N. A. Gippius, V. D. Kulakovskii, and S. G. Tikhodeev, J. Exp. Theor. Phys. 104, 715 (2007).

[22] A. A. Demenev, A. A. Shchekin, A. V. Larionov, S. S. Gavrilov, V. D. Kulakovskii, N. A. Gippius, and S. G. Tikhodeev, Phys. Rev. Lett. 101, 136401 (2008).

[23] D. N. Krizhanovskii, S. S. Gavrilov, A. P. D. Love, D. Sanvitto, N. A. Gippius, S. G. Tikhodeev, V. D. Kulakovskii, D. M. Whittaker, M. S. Skolnick, and J. S. Roberts, Phys. Rev. B 77, 115336 (2008).

[24] M. Sich, D. N. Krizhanovskii, M. S. Skolnick, A. V. Gorbach, R. Hartley, D. V. Skryabin, E. A. Cerda-Mendez, K. Biermann, R. Hey, and P. V. Santos, Nat. Photonics 6, 50 (2012).

[25] M. Wouters and I. Carusotto, Phys. Rev. B 75, 075332 (2007).

[26] R. Cerna, Y. Léger, T. K. Paraïso, M. Wouters, F. MorierGenoud, M. T. Portella-Oberli, and B. Deveaud, Nat. Commun. 4, 2008 (2013),

[27] S. S. Gavrilov and N. A. Gippius, Phys. Rev. B 86, 085317 (2012).

[28] S. S. Gavrilov, A. V. Sekretenko, S. I. Novikov, C. Schneider, S. Höfling, M. Kamp, A. Forchel, and V. D. Kulakovskii, Appl. Phys. Lett. 102, 011104 (2013).

[29] S. S. Gavrilov, A. V. Sekretenko, N. A. Gippius, C. Schneider, S. Höfling, M. Kamp, A. Forchel, and V. D. Kulakovskii, Phys. Rev. B 87, 201303 (2013).

[30] S. S. Gavrilov, Phys. Rev. B 90, 205303 (2014).

[31] S. S. Gavrilov, S. I. Novikov, V. D. Kulakovskii, N. A. Gippius, A. A. Chernov, and S. G. Tikhodeev, JETP Lett. 101, 7 (2015).

[32] S. S. Gavrilov, A. S. Brichkin, S. I. Novikov, S. Höfling, C. Schneider, M. Kamp, A. Forchel, and V. D. Kulakovskii, Phys. Rev. B 90, 235309 (2014).

[33] D. D. Solnyshkov, I. A. Shelykh, N. A. Gippius, A. V. Kavokin, and G. Malpuech, Phys. Rev. B 77, 045314 (2008).

[34] P. G. Savvidis, C. Ciuti, J. J. Baumberg, D. M. Whittaker, M. S. Skolnick, and J. S. Roberts, Phys. Rev. B 64, 075311 (2001).

[35] A. I. Tartakovskii, D. N. Krizhanovskii, D. A. Kurysh, V. D. Kulakovskii, M. S. Skolnick, and J. S. Roberts, Phys. Rev. B 65, 081308(R) (2002).

[36] N. Bogolubov, Izv. Akad. Nauk SSSR, Ser. Fiz. 11, 77 (1947) [J. Phys. (USSR) 11, 23 (1947)].

[37] A. A. Abrikosov, L. P. Gorkov, and I. E. Dzyaloshinski, Methods of the Quantum Field Theory in Statistical Physics (Dover, New York, 1977). 